\title{
Hybrid quantum/classical study of ICN in an Ar matrix: Photofragmentation and cage exit
}

\author{
S. Fernandez Alberti and J. Echave \\ Universidad Nacional de Quilmes, S. Peña 180, 1876 Bernal, Argentina \\ V. Engel \\ Institut für Physikalische Chemie, Universität Würzburg, Am Hubland, D-97074 Würzburg, Germany \\ N. Halberstadt and J. A. Beswick \\ IRSAMC, Université Paul Sabatier, 118 route de Narbonne, 31062 Toulouse, France
}

(Received 16 February 2000; accepted 17 April 2000)

\begin{abstract}
The $\widetilde{A}$ continuum photoexcitation of ICN in an Ar matrix is studied using an implementation of the molecular dynamics with quantum transitions method of Tully. Five excited electronic potential energy surfaces of the ICN molecule, ${ }^{3} \Pi_{0+},{ }^{1} \Pi_{1}\left(A^{\prime}, A^{\prime \prime}\right),{ }^{3} \Pi_{1}\left(A^{\prime}, A^{\prime \prime}\right)$, as well as its ground state, are included in these calculations. The couplings between electronic states at large I-CN internuclear distances are modeled using a diatomic in molecules treatment of the mixing of the different spin-orbit states of iodine induced by the Ar atoms. The electronic motion, as well as the I-CN distance and the corresponding bending angle, are treated quantum mechanically using wave-packet techniques. The rotation and translation of the ICN molecule in the Ar cage are treated classically, as well as the motion of the Ar atoms. In contrast with previous calculations, in which all nuclear degrees of freedom were treated classically, we found a $2 \%$ of $\mathrm{CN}$ cage exit during the first 0.5 ps of the dynamics. (c) 2000 American Institute of Physics. [S0021-9606(00)00427-X]
\end{abstract}

\section{INTRODUCTION}

The photodissociation of small molecules in rare gas matrices has been the subject of several experimental ${ }^{1-9}$ and theoretical studies. ${ }^{10-20}$ This process is a prototype of reaction dynamics in the condensed phase. The ICN molecule has been largely studied in the gas phase as a model of direct photodissociation, where the presence of a strong nonadiabatic coupling between two excited electronic potential energy surfaces (PES) determines the final state distributions of dissociation fragments. ${ }^{21-53}$

The excitation of ICN into the $\widetilde{A}$ continuum leads, in the gas phase, to dissociation into two final channels ${ }^{46,41,22,38,37}$

$$
\begin{aligned}
& \operatorname{ICN}\left(X^{1} \Sigma^{+}\right)+h \nu(3.5-5 \mathrm{eV}) \\
& \quad \rightarrow \mathrm{ICN}^{*}\left({ }^{3} \Pi_{1},{ }^{3} \Pi_{0^{+}},{ }^{1} \Pi_{1}\right)\left\{\begin{array}{l}
\left.\mathrm{I}^{2} P_{3 / 2}\right)+\mathrm{CN}\left(X^{2} \Sigma^{+}\right) \\
\mathrm{I}^{*}\left({ }^{2} P_{1 / 2}\right)+\mathrm{CN}\left(X^{2} \Sigma^{+}\right)
\end{array}\right.
\end{aligned}
$$

There are at least three excited electronic states contributing to the photodissociation dynamics in this energy range (see Fig. 1). Two of them $\left({ }^{3} \Pi_{1}\right.$ and $\left.{ }^{1} \Pi_{1}\right)$ are doubly degenerate in the linear configuration. This degeneracy is broken in the $C_{S}$ symmetry group of the bent molecule. Thus, six coupled PES $\left({ }^{3} \Pi_{1}\left(A^{\prime}, A^{\prime \prime}\right),{ }^{3} \Pi_{0^{+}},{ }^{1} \Pi_{1}\left(A^{\prime}, A^{\prime \prime}\right), X^{1} \Sigma^{+}\right)$were included in the calculations. Ab initio PES were calculated by Morokuma and co-workers in order to describe this process. ${ }^{54,55}$

To model the population transfers between different ICN electronic surfaces, the nonadiabatic couplings are needed. Two of the PES of ICN $\left({ }^{3} \Pi_{0^{+}}\right.$and $\left.{ }^{1} \Pi_{1}\right)$ present a conical intersection at short $\mathrm{I}-\mathrm{CN}$ distances that exists both in the gas phase and the condensed phase. In the latter, however, the environment induces additional couplings which are particularly efficient at large internuclear distances between the two fragments.

The photofragmentation of ICN excited in the region of the $\widetilde{A}$ continuum involves very strong vibronic couplings. Therefore, a treatment of the electronic and nuclear intramolecular vibrational states with the same level of accuracy is required. This is why, in this work, we present a model for ICN in an Ar matrix in which, in addition to the electronic dynamics, the internal vibrational degrees of freedom of ICN are treated quantum mechanically. We utilize an extended implementation of the MDQT (molecular dynamics with quantum transitions) method ${ }^{56,57}$ to study this model. While the quantum nuclear coordinates are propagated as coupled wave packets on the six electronic PES, the classical variables evolve on a single electronic PES and they can hop from one surface to another with a probability given by the MDQT method.

It is important to mention two features of the ICN photofragmentation in an Ar matrix. First, the fact that there is a first direct collision between $\mathrm{CN}$ and one of the Ar atoms, as has been shown in previous calculations. ${ }^{58}$ This strong localized collision does not allow us to treat the Ar matrix as a bath modeled by Gaussians where an harmonic motion of Ar atoms is required. ${ }^{59-61}$ Second, the couplings between the ICN electronic states at large I-CN distances are spatially delocalized in a wide range of the ICN coordinates. This makes inappropriate the use of techniques that are shown to be useful for multidimensional systems when 


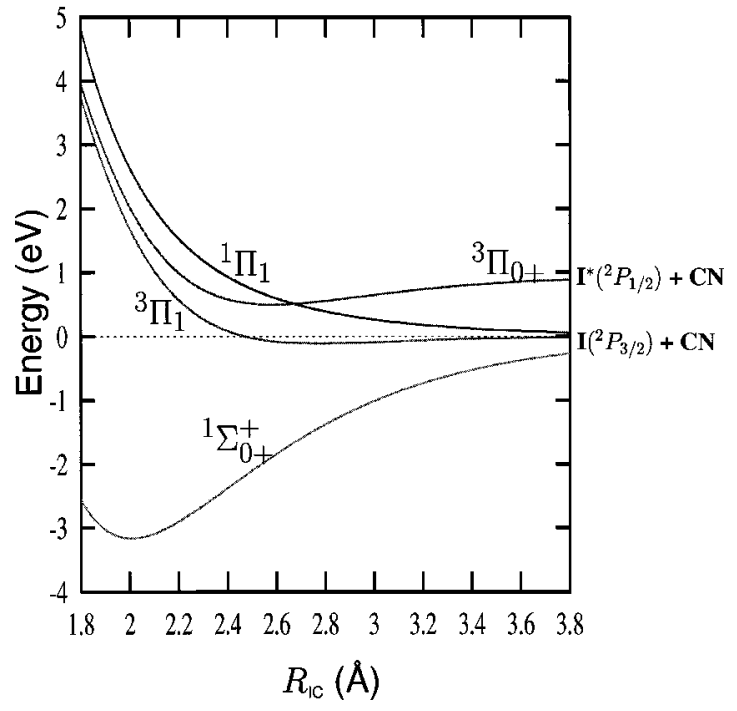

FIG. 1. Potential energy curves for collinear ICN.

the regions of nonadiabatic coupling are spatially localized. ${ }^{62,63}$

In the condensed phase, the ICN photodissociation dynamics has been studied previously by several authors. ${ }^{64-70,58}$ In the case of ICN embedded in a cryogenic Ar matrix, previous molecular dynamics simulations have found no cage exit of the fragments. ${ }^{67,58}$ This does not agree with recent experimental results ${ }^{71}$ which have found a small but detectable amount of cage exit. The aim of this work was to study the effect of a quantum treatment of the ICN vibrational coordinates in the first stages of the photodissociation dynamics. In particular, we were interested in checking whether such a treatment could reproduce the experimentally observed cage exit of $\mathrm{CN}$.

\section{METHODOLOGY}

\section{A. Coupled wave-packet MDQT}

The hybrid quantal/classical approach consists of dividing the total number of degrees of freedom into a quantum part and a classical part. In the present work we use the molecular dynamics with quantum transitions (MDQT) $)^{56,57}$ method of Tully, in which the electronic degrees of freedom $\mathbf{x}$ and some of the nuclear degrees of freedom $\mathbf{X}_{\mathbf{q}}$ are described by coupled wave packets, while the other nuclear coordinates $\mathbf{X}_{\mathbf{c l}}$ are treated classically.

In this work the wave function $\Phi\left(\mathbf{X}_{\mathbf{q}}, \mathbf{x}, t\right)$ that describes the quantum-mechanical state at time $t$ is written as

$$
\begin{aligned}
\Phi\left(\mathbf{X}_{\mathbf{q}}, \mathbf{x}, t\right) & =\sum_{j}^{n} \Phi_{j}\left(\mathbf{X}_{\mathbf{q}}, \mathbf{x}, t\right) \\
& =\sum_{j}^{n} \psi_{j}\left(\mathbf{X}_{\mathbf{q}}, t\right) \phi_{j}\left(\mathbf{x} ; \mathbf{X}_{\mathbf{q}}, \mathbf{X}_{\mathbf{c l}}(t)\right),
\end{aligned}
$$

the $\psi_{j}\left(\mathbf{X}_{\mathbf{q}}, t\right)$ being nuclear wave packets associated to the diabatic electronic states $\phi_{j}\left(\mathbf{x} ; \mathbf{X}_{\mathbf{q}}, \mathbf{X}_{\mathbf{c l}}(t)\right)$. These wave packets are propagated in time by integrating the coupled wave-packet equations

$$
i \hbar \frac{\partial \psi_{i}\left(\mathbf{X}_{\mathbf{q}}, t\right)}{\partial t}=\sum_{j}^{n} H_{q, i j}\left(\mathbf{X}_{\mathbf{q}}, \mathbf{X}_{\mathbf{c l}}(t)\right) \psi_{j}\left(\mathbf{X}_{\mathbf{q}}, t\right),
$$

where

$$
H_{q, i j}\left(\mathbf{X}_{\mathbf{q}}, \mathbf{X}_{\mathbf{c l}}(t)\right)=K_{q}\left(\mathbf{X}_{\mathbf{q}}\right) \delta_{i j}+V_{i j}\left(\mathbf{X}_{\mathbf{q}}, \mathbf{X}_{\mathbf{c l}}(t)\right),
$$

with $K_{q}\left(\mathbf{X}_{\mathbf{q}}\right)$ being the kinetic energy operator associated to the quantum nuclear coordinates $\mathbf{X}_{\mathbf{q}}$ and $V_{i j}\left(\mathbf{X}_{\mathbf{q}}, \mathbf{X}_{\mathbf{c l}}(t)\right)$ the corresponding diabatic potential matrix elements.

The classical trajectories are run on a single electronic state $\Phi_{k}(t)=\Phi_{k}\left(\mathbf{X}_{\mathbf{q}}, \boldsymbol{x}, t\right)$ [see Eq. (2)] according to the Hamilton's equations of motion

$$
\begin{aligned}
& \dot{\mathbf{X}}_{\mathbf{c l}}=\left\langle\Phi_{k}(t)\left|\frac{\partial H}{\partial \mathbf{P}_{\mathbf{X}_{\mathbf{c l}}}}\right| \Phi_{k}(t)\right\rangle /\left\langle\Phi_{k}(t) \mid \Phi_{k}(t)\right\rangle, \\
& \dot{\mathbf{P}}_{\mathbf{X}_{\mathbf{c l}}}=-\left\langle\Phi_{k}(t)\left|\frac{\partial H}{\partial \mathbf{X}_{\mathbf{c l}}}\right| \Phi_{k}(t)\right\rangle /\left\langle\Phi_{k}(t) \mid \Phi_{k}(t)\right\rangle,
\end{aligned}
$$

where the brackets imply the integration over the electronic $(\mathbf{x})$ and nuclear quantum variables $\left(\mathbf{X}_{\mathbf{q}}\right)$ and $H$ $=H\left(\mathbf{x}, \mathbf{X}_{\mathbf{q}}, \mathbf{X}_{\mathbf{c l}}\right)$ is the total Hamiltonian.

At each time step the classical trajectory can hop from one surface to another. The hopping probability from state $\Phi_{i}$ to $\Phi_{j}$ was calculated using ${ }^{56,57}$

$g_{i \rightarrow j}(t)$

$$
=\frac{\Delta t}{\hbar} \frac{2 \operatorname{Im}\left\{\left\langle\psi_{i}\left(\mathbf{X}_{\mathbf{q}}, t\right)\left|V_{i j}\left(\mathbf{X}_{\mathbf{q}}, \mathbf{X}_{\mathbf{c l}}(t)\right)\right| \psi_{j}\left(\mathbf{X}_{\mathbf{q}}, t\right)\right\rangle\right\}}{\left\langle\psi_{i}\left(\mathbf{X}_{\mathbf{q}}, t\right) \mid \psi_{i}\left(\mathbf{X}_{\mathbf{q}}, t\right)\right\rangle},
$$

where $V_{i j}\left(\mathbf{X}_{\mathbf{q}}, \mathbf{X}_{\mathbf{c l}}(t)\right)$ is the coupling matrix element between the electronic states $i$ and $j$. A velocity adjustment during a hop is made according to ${ }^{72}$

$$
\mathbf{P}_{\mathbf{X}_{\mathbf{c l}}^{\prime}}=\mathbf{P}_{\mathbf{X}_{\mathbf{c l}}}-\gamma \nabla_{\mathbf{X}_{\mathbf{c l}}}\left\langle\psi_{i}\left(\mathbf{X}_{\mathbf{q}}, t\right)\left|V_{i j}\left(\mathbf{X}_{\mathbf{q}}, \mathbf{X}_{\mathbf{c l}}(t)\right)\right| \psi_{j}\left(\mathbf{X}_{\mathbf{q}}, t\right)\right\rangle,
$$

where $\mathbf{P}_{\mathbf{X}_{\mathbf{c l}}^{\prime}}$ and $\mathbf{P}_{\mathbf{X}_{\mathbf{c l}}}$ are the classical momenta after and before the transition, respectively. The value of $\gamma$ is obtained by imposing energy conservation. In addition, if $\left.\left\langle\psi_{j}\left(\mathbf{X}_{\mathbf{q}}, t\right)\left|V_{j j}\left(\mathbf{X}_{\mathbf{q}}, \mathbf{X}_{\mathbf{c l}}(t)\right)\right| \psi_{j}\left(\mathbf{X}_{\mathbf{q}}, t\right)\right\rangle\right\rangle\left\langle\psi_{i}\left(\mathbf{X}_{\mathbf{q}}, t\right)\right|$ $V_{i i}\left(\mathbf{X}_{\mathbf{q}}, \mathbf{X}_{\mathbf{c l}}(t)\right)\left|\psi_{i}\left(\mathbf{X}_{\mathbf{q}}, t\right)\right\rangle$, and if there is not enough kinetic energy to ensure energy conservation, the hop is forbidden.

\section{B. Application to ICN-Ar}

To treat the ICN molecule, we used the Jacobi vector $\mathbf{R}$, joining I to the $\mathrm{CN}$ center of mass, $\mathbf{r}$, the internuclear vector of $\mathrm{CN}$, and $\mathbf{R}_{\mathbf{C M}}$, corresponding to the center of mass of ICN. In the present work, the Jacobi I-CN distance $R$ and the bending angle $\theta$ between $\mathbf{R}$ and $\mathbf{r}$ were treated quantum mechanically. The $\mathrm{C}-\mathrm{N}$ internuclear distance $r$ was fixed at its equilibrium position $r_{\text {eq }}=2.2372$ a.u. Previous calculations have shown that this coordinate does not play an important role in the dissociation process. ${ }^{40,58}$ The coupled wave packets $\psi_{j}(R, \theta, t)$ satisfy Eqs. (3) and (4) with

$$
\begin{aligned}
K_{q}(R, \theta)= & -\frac{\hbar^{2}}{2 \mu_{\mathrm{I}, \mathrm{CN}}} \frac{\partial^{2}}{\partial R^{2}}-\hbar^{2}\left(\frac{1}{2 \mu_{\mathrm{I}, \mathrm{CN}} R^{2}}\right. \\
& \left.+\frac{1}{2 \mu_{\mathrm{CN}} r_{\mathrm{eq}}^{2}}\right) \frac{1}{\sin \theta} \frac{\partial}{\partial \theta} \sin \theta \frac{\partial}{\partial \theta},
\end{aligned}
$$




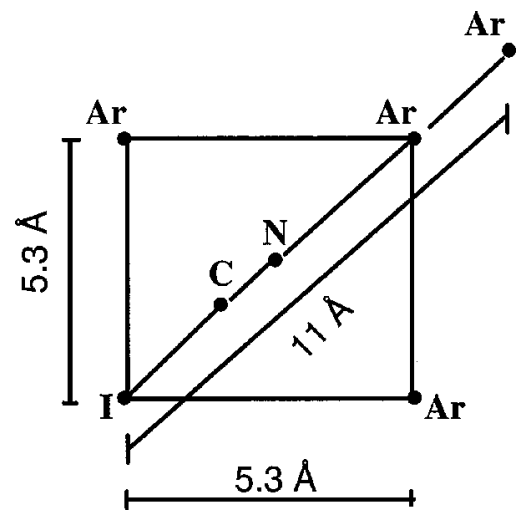

FIG. 2. The ICN molecule at its initial disubstitutional position in the central unit cell of the Ar matrix ( $x^{\prime}, y^{\prime}$ plane).

where $\quad \mu_{\mathrm{CN}}=m_{\mathrm{C}} m_{\mathrm{N}} /\left(m_{\mathrm{C}}+m_{\mathrm{N}}\right), \quad \mu_{\mathrm{I}, \mathrm{CN}}=m_{\mathrm{I}}\left(m_{\mathrm{C}}+m_{\mathrm{N}}\right) /$ $\left(m_{\mathrm{I}}+m_{\mathrm{C}}+m_{\mathrm{N}}\right)$, and

$$
V_{i j}(R, \theta, t)=V_{i j}^{\mathrm{ICN}}(R, \theta)+V_{i j}^{\mathrm{int}}(R, \theta, t),
$$

where $V_{i j}^{\mathrm{ICN}}(R, \theta)$ are the electronic diabatic potential matrix elements, and $V_{i j}^{\text {int }}(R, \theta, t)$ the corresponding matrix elements of the $\mathrm{Ar}-\mathrm{ICN}$ interaction potential (although not explicitly written, they are also functions of all Ar coordinates).

In order to simulate the photoexcitation, we used the following initial conditions for the wave-packet components:

$$
\psi_{f}(R, \theta, t=0)=\mu_{f i}(R, \theta) \psi_{i}(R, \theta), \quad f \neq i,
$$

where $i$ is the electronic ground state, $\psi_{i}(R, \theta)$ is the corresponding ground vibrational state, and $\mu_{f i}(R, \theta)$ are the transition dipole moments.

The other nuclear degrees of freedom, that is, the three Euler's angles specifying the orientation of the ICN molecule, the ICN center of mass coordinates, and the argon Cartesian coordinates, were all treated classically. We solved Eqs. (5) with

$$
\begin{aligned}
H= & \left(\frac{1}{2 \mu_{\mathrm{I}, \mathrm{CN}} R^{2}}+\frac{1}{2 \mu_{\mathrm{CN}} r_{\mathrm{eq}}^{2}}\right) \frac{P_{\phi^{\prime}}^{2}}{\sin ^{2} \theta}+\frac{\mathbf{J}^{2}}{2 \mu_{\mathrm{I}, \mathrm{CN}}}-\frac{\mathbf{j} \cdot \mathbf{J}}{\mu_{\mathrm{I}, \mathrm{CN}} R^{2}} \\
& +T^{\mathrm{Ar}}+T_{\mathrm{CM}}^{\mathrm{ICN}}+V^{\mathrm{int}}+V^{\mathrm{Ar}-\mathrm{Ar}}
\end{aligned}
$$

where

$$
T_{\mathrm{CM}}^{\mathrm{ICN}}=\frac{1}{2 M}\left(P_{X_{\mathrm{CM}}}^{2}+P_{Y_{\mathrm{CM}}}^{2}+P_{Z_{\mathrm{CM}}}^{2}\right)
$$

and

$$
T^{\mathrm{Ar}}=\frac{1}{2 m_{\mathrm{Ar}}} \sum_{\alpha}^{n_{\mathrm{Ar}}}\left(P_{X_{\alpha}}^{2}+P_{Y_{\alpha}}^{2}+P_{Z_{\alpha}}^{2}\right)
$$

are the kinetic energies of ICN center of mass and the Ar atoms, respectively. $V^{\text {int }}$ is the $\mathrm{ICN}-\mathrm{Ar}$ interaction potential and $V^{\mathrm{Ar}-\mathrm{Ar}}$ is the $\mathrm{Ar}-\mathrm{Ar}$ potential.

The $\mathbf{J}$ and $\mathbf{j}$ are the angular momentum of ICN and $\mathrm{CN}$, respectively. At this point it is important to note that, while the expression for $\mathbf{J}^{\mathbf{2}}$ is completely classical, the Coriolis term $\mathbf{j} \cdot \mathbf{J}$ is a mixed quantum/classical term (see the Appendix).

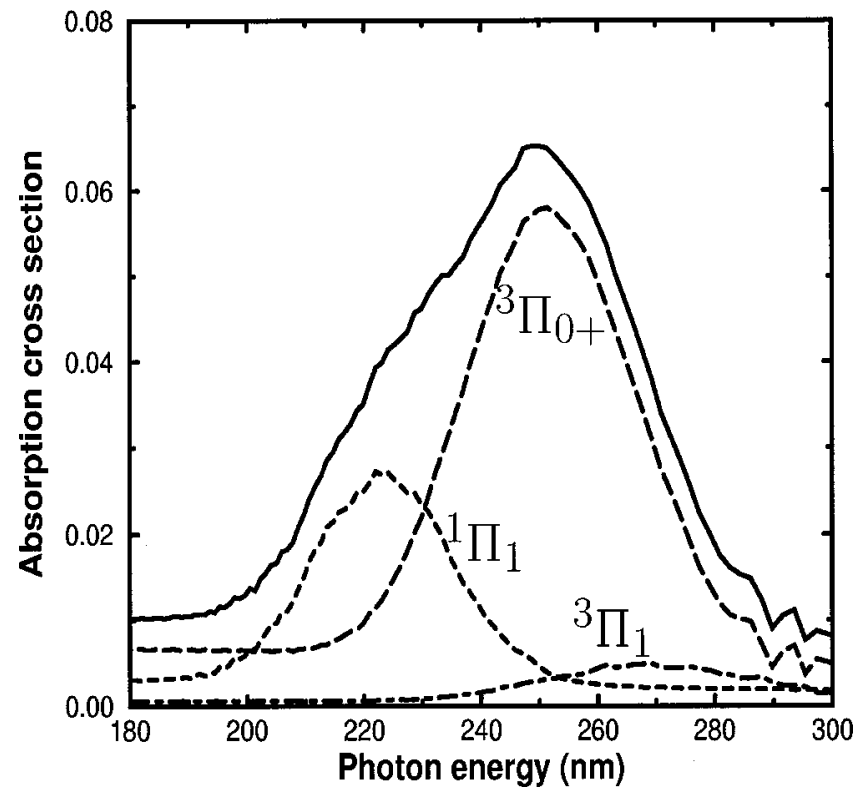

FIG. 3. Calculated absorption spectrum of ICN at Ar.

\section{Potentials and couplings}

The ICN electronic potential energy surfaces $\left[{ }^{3} \Pi_{1}\left(A^{\prime}, A^{\prime \prime}\right),{ }^{3} \Pi_{0^{+}},{ }^{1} \Pi_{1}\left(A^{\prime}, A^{\prime \prime}\right), X^{1} \Sigma^{+}\right]$, interstate couplings at short I-CN distances, and transition moments, as well as the $\mathrm{Ar}-\mathrm{ICN}$ and $\mathrm{Ar}-\mathrm{Ar}$ interaction potentials used in this work, were taken from Morokuma and co-workers. ${ }^{55,70}$

Interstate couplings at large I-CN distances were obtained from a diatomic in molecules (DIM) treatment ${ }^{73-76,12}$ of the mixing between different spin-orbit states of iodine induced by the argon atoms, with the potential parameters determined by Neumark and collaborators. ${ }^{77}$ The procedure to obtain ICN states and couplings at large I-CN distances is similar to that used in previous work. ${ }^{76,12,58}$ Briefly, the different states correlating with $I\left({ }^{2} P_{3 / 2}\right)+\mathrm{CN}\left(X^{2} \Sigma^{+}\right)$ $+\operatorname{Ar}\left({ }^{1} S_{0}\right)$ are written as $|J, \Omega\rangle$, where $J$ and $\Omega$ stand for the total electronic angular momentum of ICN and its projection onto the quantization axis, respectively. These states are expressed as combinations of uncoupled states $\left|J_{\mathrm{I}}, \Omega_{\mathrm{I}}\right\rangle\left|J_{\mathrm{CN}}, \Omega_{\mathrm{CN}}\right\rangle$. The effects of the $\mathrm{CN}$ orientation at large $\mathrm{CN}-\mathrm{I}$ distances are neglected. Then, the $\left|J_{\mathrm{I}}, \Omega_{\mathrm{I}}\right\rangle$ and $\left|J_{\mathrm{CN}}, \Omega_{\mathrm{CN}}\right\rangle$ states are written in the $\mathrm{L}-\mathrm{S}$ representation. The procedure was applied, as in previous work, ${ }^{58}$ only for distances large enough so that the value of the attractive component of the ground-state potential is less than the spin-orbit constant of the I atom. Therefore, the uncoupled representation of the ICN molecule is valid.

\section{Numerical details}

We have considered a system composed of ICN and 254 Ar atoms using the minimum image convention ${ }^{78}$ at the boundaries. According to previous work, this number of $\mathrm{Ar}$ atoms corresponds to a box large enough to avoid size effects for times up to a few picoseconds. ${ }^{67}$

The propagation of the coupled nuclear wave packets was performed using the split-operator technique (SOT). ${ }^{79-81}$ A discrete variable representation (DVR) of Legendre poly- 

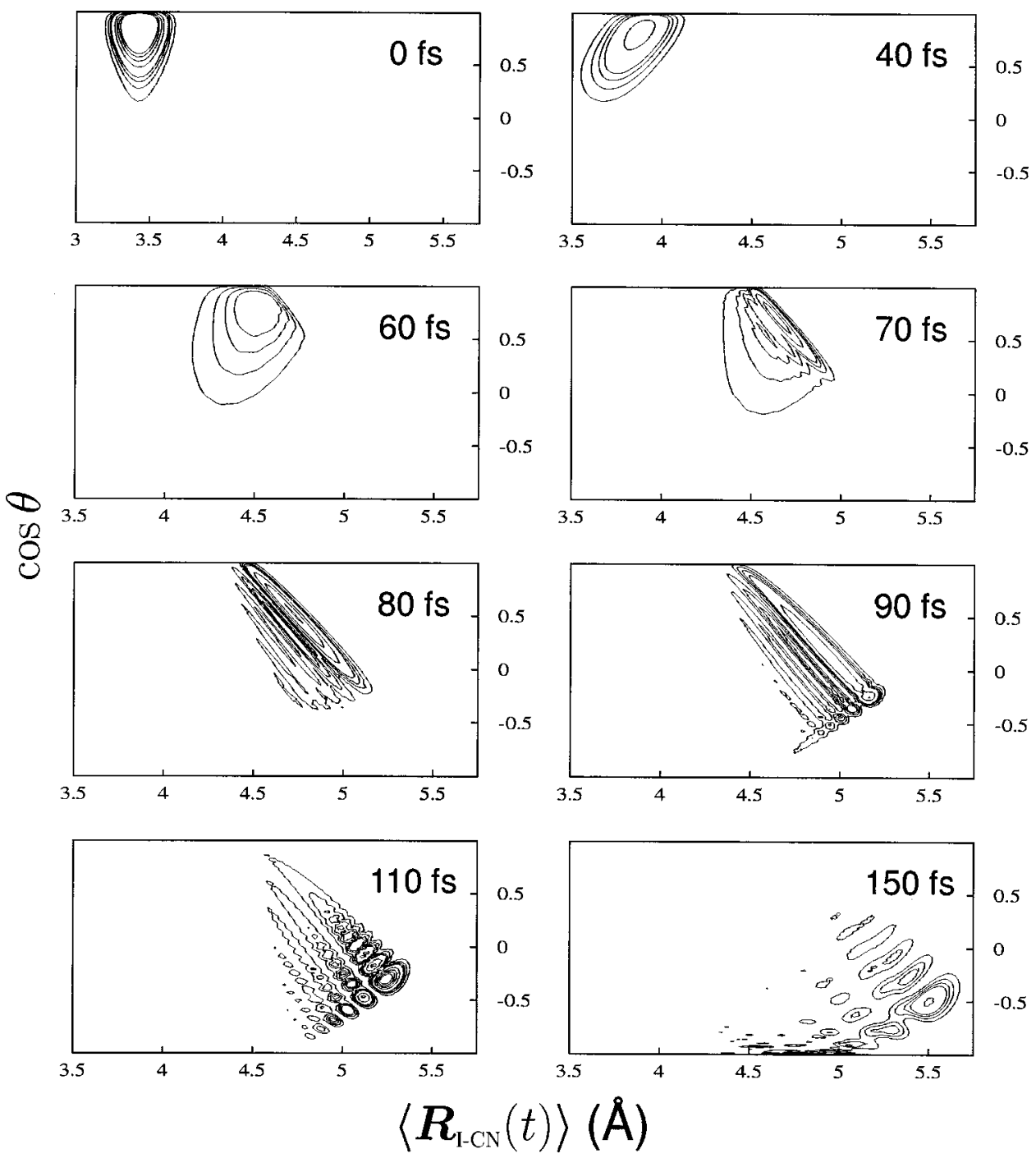

FIG. 4. Time evolution of the nuclear wave packet associated to the ${ }^{3} \Pi_{0^{+}}$ electronic surface for a typical classical trajectory.

nomials was used for the angular coordinate $(\theta) .{ }^{82}$ We used 80 points in the $\theta$ coordinate and a grid of 512 points in the $R$ coordinate. The spacing in $R$ was 0.028 a.u. The time step $\delta t$ for the quantal part of the system was $0.005 \mathrm{fs}$, whereas the classical time step was $\Delta t=100 \delta t$. The Hamming predictor-corrector algorithm was used in the propagation of classical coordinates.

First, a hybrid quantum/classical simulation of 25 ps was run in the ground state with the ICN molecule placed initially in a disubstitutional site as sugested by Gerber et al. ${ }^{67}$ (Fig. 2). The initial values of Euler's angles were: $\theta_{R}^{\prime}$ $=\pi / 4, \phi_{R}^{\prime}=\pi / 2$ and $\phi^{\prime}=0$. The initial ICN wave function we used in this ground-state simulation was a stationary wave function in the $\nu=0$ vibrational state of the ICN ground electronic surface obtained by imaginary time propagation. The system was allowed to equilibrate during 5 ps arriving to a final temperature of $\sim 4 \mathrm{~K}$ (average kinetic energy). The last 20 ps were used to collect, every $250 \mathrm{fs}$, a set of initial wave functions and classical positions and momenta for the subsequent photodissociation dynamics.

To avoid problems of nonphysical reflexions in the limits of the grid, we absorbed the wave packet using the splitting method developed by Pernot and Lester. ${ }^{83}$ The absorp- tion region was $10.3 \AA<R<11.4 \AA$. It is important to stress that this interval is outside the radius of first neighbors in the matrix (see Fig. 2). Thus, the calculated cumulative absorption probability can be interpreted as the probability for the ICN molecule to escape the solvent cage.

A total of 40 photodissociation trajectories of 500 fs was propagated in order to obtain reproducible results. While the quantum treatment of ICN internal degrees of freedom is much more time consuming than our previous calculations, in which they were treated classically, the Ar atom oscillations and the Euler's angles displacements are found to be movements of small amplitude and therefore well described with a minimum number of trajectories.

\section{RESULTS AND DISCUSSION}

The ICN absorption spectrum calculated from the autocorrelation function after $500 \mathrm{fs}$ of dynamics is shown in Fig. 3. ${ }^{84}$ The weakness of the ICN-Ar van der Waals interactions and the spread of the wave packet during the first collision with the cage (after it reached the dissociation limit) leads to a structureless absorption spectrum very similar to previous results in the gas phase. ${ }^{40}$ 

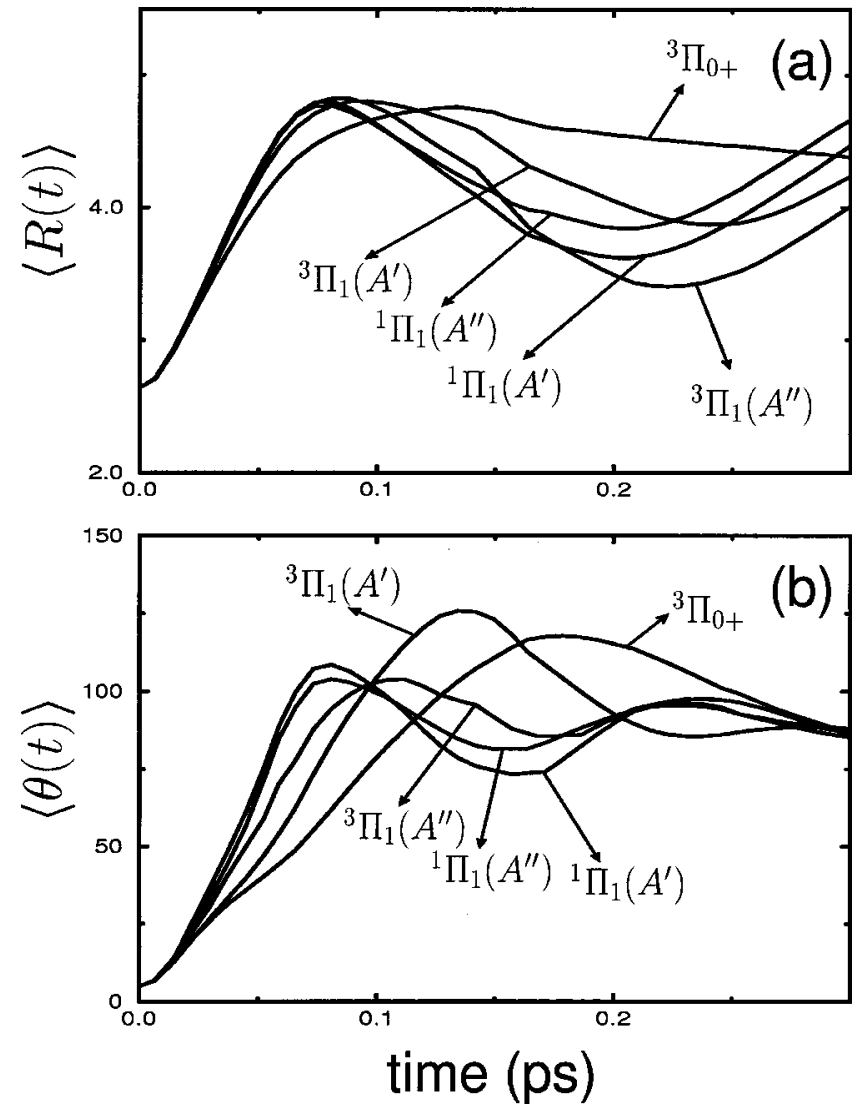

FIG. 5. Average over all the trajectories of (a) $\langle R(t)\rangle$, (b) $\langle\theta(t)\rangle$.

Similar to the gas phase dynamics, as soon as the ICN molecule is excited to the repulsive electronic PESs of the $\widetilde{A}$ band, it starts moving out along the dissociative coordinate $R$. In Fig. 4, we show the time evolution of the wave-packet component on the ${ }^{3} \Pi_{0^{+}}$surface. We see that the component of the wave packet corresponding to a linear configuration $(\theta=0)$ will be the first to interact strongly with the argon matrix (at $\sim 60 \mathrm{fs}$ ). This is because the kinetic energy in excess after the excitation is localized along the radial coordinate. During this first collision with the cage, the collinear part of the wave packet bounces (Fig. 4, 60-70 fs), and starts to overlap with the wave-packet portions that are arriving more slowly in a nonlinear configuration (Fig. 4, 70-80 fs). Most of the wave packet bounces with angles close to $\theta$ $=\pi / 2$, and $R \sim 5.5 \AA$ (Fig. 4,90 fs). This distance corresponds roughly to the collision distance for the linear configuration $(\sim 4.5 \AA$ ) plus the $\mathrm{C}-\mathrm{N}$ bond distance $(1.2 \AA$ ) (see Fig. 2). After the collision, interferences between the part of the wave packet that has already been reflected by the argon matrix and the one that is arriving to the cage boundary are clearly seen (Fig. 4, $110 \mathrm{fs}$ ).

At longer times, due to the symmetry of the wave function around $\theta=\pi$, the part of the wave packet that approaches $\pi$ interferes with the one that moves away from $\pi$ (Fig. 4, $150 \mathrm{fs}$ ). It is important to stress that the $\mathbf{R}$ vector remains in the plane of the molecule throughout the simulation, while the $\mathbf{r}$ vector starts oscillating around that plane after the collision. Thus, the process described in Fig. 4 takes

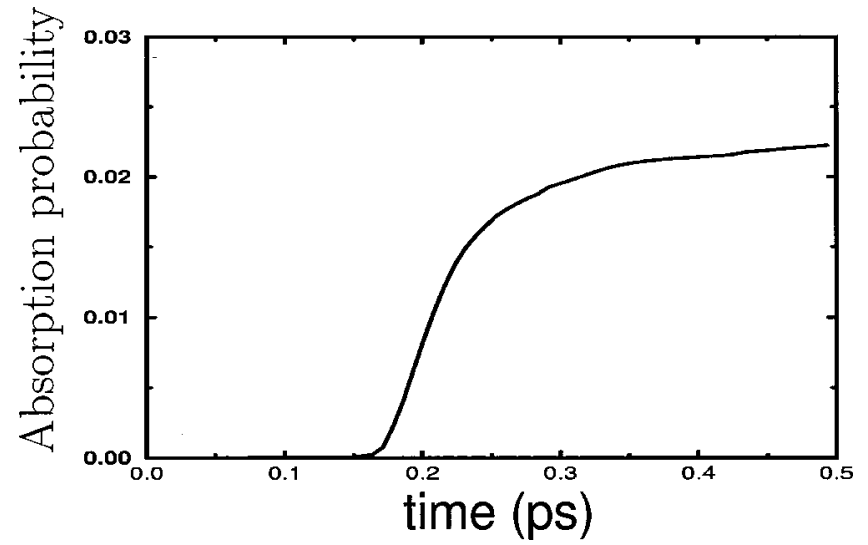

FIG. 6. Normalized absorption probability in the [10.3 $\mathrm{A}-11.4 \AA$ ] region.

place mainly on the $y^{\prime} z^{\prime}$ plane of the $\left(x^{\prime}, y^{\prime}, z^{\prime}\right)$ fixed frame of the Ar matrix (see Fig. 2).

In Figs. 5(a) and 5(b) we show the averages of $R$ and $\theta$ over the trajectories for each of the nuclear wave-packet components $\Phi_{k}\left(\mathbf{X}_{\mathbf{q}}, \mathbf{x}, t\right)$. It is seen that the motion of the different $\Phi_{k}\left(\mathbf{X}_{\mathbf{q}}, \mathbf{x}, t\right)$ components dephases rapidly after 50 fs. This shows clearly that a mean-field treatment of this problem would not be appropriate, thus validating our surface-hopping approach.

The most important result of these calculations is that there is about $2 \%$ of permanent dissociation of ICN. This is displayed in Fig. 6, where we represent the time dependence of the average cumulative probability of cage exit. In the present calculations, we used the absorption function:

$$
f_{a}=\left\{\begin{array}{l}
e^{-a(R-b)^{2}} \quad \text { if } \quad R>10.3 \AA \\
1 \quad \text { if } \quad R<10.3 \AA .
\end{array}\right.
$$

Results were converged with respect to small changes of the absorption region and absorption function parameters $a$ and $b$. The slow convergence in time of the average absorption probability (Fig. 6) as well as the fact that the average of the wave packet bounces with $R \sim 5 \AA$ (Figs. 4 and 5), a much smaller distance than the grid boundaries, indicate that Fig. 6 is not an artifact. This result is in good agreement with experimental results of Chergui et al. ${ }^{71}$ and represents the main difference with previous theoretical results ${ }^{67,58}$ in which the internal vibrational degrees of freedom of ICN were treated classically.

The process of cage exit takes place during the first 400 fs of the photodissociation dynamics. After that time, the cumulative probability of cage exit reaches its final constant value. In order to understand this behavior, we have represented the time dependence of the average radial kinetic energy of ICN (Fig. 7). We can see that, after the first collision of the $\mathrm{CN}$ fragment with the Ar cage, the radial kinetic energy of ICN is much smaller than before the collision. Therefore, any cage exit has to take place during this first collision. The time dependence of the average ICN potential energy, $V^{\mathrm{ICN}}+V^{\text {int }}$, is shown in Fig. 7. It presents a maximum of about $1 \mathrm{eV}$ at the time of the first $\mathrm{CN}$-cage collision $(\sim 60 \mathrm{fs})$. Subsequently, $\mathrm{CN}$ is much slower and it never 


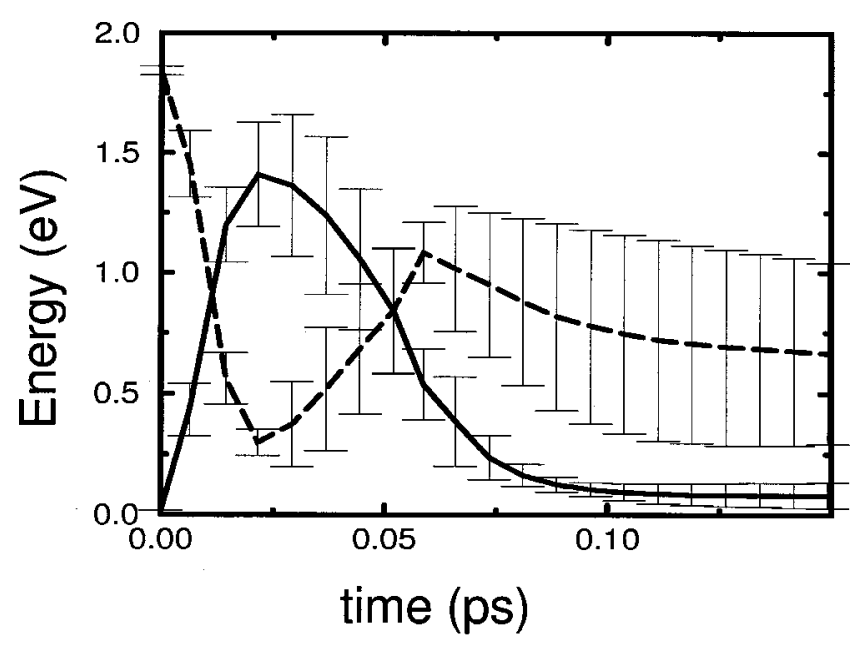

FIG. 7. Time dependence of averaged values of potential $\langle V(t)\rangle(---)$ and radial kinetic energies $\langle K(t)\rangle(-)$. The bars represent the dispersion of the results.

gets as close to the cage as in the first collision. It interacts with several Ar atoms, experiencing an average repulsive potential that changes slowly in time.

\section{CONCLUSIONS}

We have studied the photodissociation dynamics of ICN in a cryogenic argon matrix using a hybrid quantum/classical technique based on the MDQT method of Tully. ${ }^{56,57} \mathrm{We}$ have introduced nuclear wave packets (one for each electronic state) describing the ICN internal vibrational coordinates $(R, \theta)$ in a matrix of $254 \operatorname{argon}$ atoms. Thus, while the ICN internal coordinates were propagated quantum mechanically in a body-fixed frame of the ICN molecule, the other degrees of freedom were propagated classically in the spacefixed frame of the matrix.

Six ICN electronic surfaces and their corresponding couplings were included in the calculations.

It is important to mention that the restricted number of trajectories that can be run in condensed phase systems is one of the principal limitations of the MDQT technique. ${ }^{56}$ An insufficient number of trajectories introduces an artificial excess of coherence between the coupled electronic states. The quantum treatment of the vibrational degrees of freedom that are more strongly involved in the fragmentation dynamics is important to overcome this limitation.

Our wave-packet calculations allow a detailed analysis of the role of the first collision with the cage and yield a probability for cage exit of the $\mathrm{CN}$ fragment of the order of $2 \%$. This result is in good agreement with the experimental results of Chergui et al. $^{71}$ and represents the main difference with previous theoretical results. ${ }^{67,58}$ Cage exit is shown to occur during the first collision of the $\mathrm{CN}$ fragment with the argon matrix. After the first collision with the cage, the residual radial kinetic energy of ICN becomes too small to allow the $\mathrm{CN}$ fragment to get close enough to the cage boundaries.

\section{ACKNOWLEDGMENTS}

This work was supported by the French-German collaborative program Procope and the Argentina-French program ECOS A97E01. We would like to thank M. Chergui, J. Helbing, and C. Meier for very helpful discussions.

\section{APPENDIX}

In the present work, the quantum Hamiltonian $H_{q}$ was written in the molecular frame of ICN $(x, y, z)$ with the $\mathbf{r}$ vector in its $x z$ plane. Nevertheless, while the nuclear quantum coordinates $R$ and $\theta$ were propagated in this reference frame, the others degrees of freedom were all propagated classically in the fixed frame of the matrix $\left(x^{\prime}, y^{\prime}, z^{\prime}\right)$.

The total angular velocity $\boldsymbol{\omega}$ at which the ICN molecular frame turns is given by the sum of angular velocity vectors that correspond to variations of the three Euler's angles

$$
\boldsymbol{\omega}=\boldsymbol{\omega}_{\theta_{R}^{\prime}}+\boldsymbol{\omega}_{\phi_{R}^{\prime}}+\boldsymbol{\omega}_{\phi^{\prime}},
$$

that are related to $\boldsymbol{\omega}$ Cartesian coordinates by ${ }^{85,86}$

$$
\begin{aligned}
& \omega_{x}=\sin \phi^{\prime} \dot{\theta}_{R}^{\prime}-\sin \theta_{R}^{\prime} \cos \phi^{\prime} \dot{\phi}_{R}^{\prime}, \\
& \omega_{y}=\cos \phi^{\prime} \dot{\theta}_{R}^{\prime}+\sin \theta_{R}^{\prime} \sin \phi^{\prime} \dot{\phi}_{R}^{\prime}, \\
& \omega_{z}=\cos \theta_{R}^{\prime} \dot{\phi}_{R}^{\prime}+\dot{\phi}^{\prime} .
\end{aligned}
$$

Besides, the rotational kinetic energy $T$ of a triatomic molecule in a molecular frame in which the $\mathbf{r}$ vector is in the $x z$ plane can be expressed as

$$
2 T=\boldsymbol{\omega} \cdot \mathbf{I} \cdot \boldsymbol{\omega}+2 I_{r} \dot{\theta} \omega_{y}+I_{r} \dot{\theta}^{2},
$$

where the $\mathbf{I}$ tensor is

$$
\mathbf{I}=\left(\begin{array}{ccc}
I_{R}+I_{r} \cos ^{2} \theta & 0 & -I_{r} \sin \theta \cos \theta \\
0 & I_{R}+I_{r} & 0 \\
-I_{r} \sin \theta \cos \theta & 0 & I_{r} \sin ^{2} \theta
\end{array}\right),
$$

with

$$
\begin{gathered}
I_{R}=\mu_{\mathrm{I}, \mathrm{CN}} R^{2}, \\
I_{r}=\mu_{\mathrm{CN}} r^{2} .
\end{gathered}
$$

Then, we can replace Eq. (A4) and (A2) in (A3) to calculate the conjugated momenta of Euler's angles

$$
\begin{aligned}
P_{\theta_{R}^{\prime}}=\frac{\partial T}{\partial \dot{\theta}_{R}^{\prime}}= & \left(I_{x x} \omega_{x}+I_{x z} \omega_{z}\right) \sin \phi^{\prime}+I_{y y} \omega_{y} \cos \phi^{\prime} \\
& +I_{r} \omega_{\theta} \cos \phi^{\prime}, \\
P_{\phi_{R}^{\prime}}=\frac{\partial T}{\partial \dot{\phi}_{R}^{\prime}}= & \left(I_{x x} \omega_{x}+I_{x z} \omega_{z}\right)\left(-\sin \theta_{R}^{\prime} \cos \phi^{\prime}\right) \\
& +I_{y y} \omega_{y} \sin \theta_{R}^{\prime} \sin \phi^{\prime}+I_{z z} \omega_{z} \cos \theta_{R}^{\prime} \\
& +I_{r} \omega_{\theta} \sin \theta^{\prime} \sin \phi^{\prime}+I x z \omega_{x} \cos \theta_{R}^{\prime},
\end{aligned}
$$




$$
\begin{aligned}
& P_{\phi^{\prime}}=\frac{\partial T}{\partial \dot{\phi}^{\prime}}=I_{z z} \omega_{z}+I_{x z} \omega_{x}, \\
& P_{\theta}=\frac{\partial T}{\partial \dot{\theta}}=I_{r}\left(\omega_{y}+\omega_{\theta}\right) .
\end{aligned}
$$

Thus, by inversion of Eq. (A6), we can obtain the components of the total angular momentum vector $\mathbf{J}$ in the molecular frame as

$$
\begin{aligned}
J_{x}= & \frac{\partial T}{\partial \omega_{x}}=I_{x x} \omega_{x}+I_{x z} \omega_{z}=P_{\theta_{R}^{\prime}} \sin \phi^{\prime}-P_{\phi_{R}^{\prime}} \frac{\cos \phi^{\prime}}{\sin \theta_{R}^{\prime}} \\
& +P_{\phi^{\prime}} \cot \theta_{R}^{\prime} \cos \phi^{\prime}, \\
J_{y}= & \frac{\partial T}{\partial \omega_{y}}=I_{y y} \omega_{y}+I_{r} \dot{\theta}=P_{\theta_{R}^{\prime}} \cos \phi^{\prime}+P_{\phi_{R}^{\prime}} \frac{\sin \phi^{\prime}}{\sin \theta_{R}^{\prime}} \\
& -P_{\phi^{\prime}} \cot \theta_{R}^{\prime} \sin \phi^{\prime}, \\
J_{z}= & \frac{\partial T}{\partial \omega_{z}}=I_{z z} \omega_{z}+I_{x z} \omega_{x}=P_{\phi^{\prime}}{ }^{\prime} .
\end{aligned}
$$

Finally, we can express $J^{2}$ as:

$$
\begin{aligned}
J^{2}= & J_{x}^{2}+J_{y}^{2}+J_{z}^{2}=P_{\theta_{R}^{\prime}}^{2}+\frac{1}{\sin ^{2} \theta_{R}^{\prime}}\left(P_{\phi_{R}^{\prime}}^{2}+P_{\phi^{\prime}}^{2}\right) \\
& -2 \frac{\cot \theta_{R}^{\prime}}{\sin \theta_{R}^{\prime}} P_{\phi^{\prime}} P_{\phi_{R}^{\prime}} .
\end{aligned}
$$

We are interested now in a classical expression of the $\mathrm{CN}$ fragment angular momentum $\mathbf{j}$ in the body-fixed frame with the $\mathbf{r}$ vector in the $x z$ plane. We know that the quantummechanical expression of its Cartesian components ${ }^{87}$ is

$$
\begin{aligned}
& j_{x}=-\frac{\hbar}{i} \cot \theta \frac{\partial}{\partial \phi^{\prime}}, \\
& j_{y}=\frac{\hbar}{i} \frac{\partial}{\partial \theta}, \\
& j_{z}=\frac{\hbar}{i} \frac{\partial}{\partial \phi^{\prime}}=J_{z} .
\end{aligned}
$$

The $\mathbf{j}$ projection on the $y$ axis is taken as zero because $\left\langle Y_{j \Omega}|\partial / \partial \theta| Y_{j \Omega}\right\rangle=0$. Then, using quantum-classical correspondences we write the classical expression of the Cartesian components of $\mathbf{j}$ in the body-fixed frame

$$
\begin{aligned}
& j_{x}=-\cot \theta P_{\phi}^{\prime}, \\
& j_{y}=0, \\
& j_{z}=P_{\phi}^{\prime} .
\end{aligned}
$$

Finally, we can obtain an expression for the Coriolis term $\mathbf{j} \cdot \mathbf{J}$ using Eqs. (A10) and (A7)

$$
\begin{aligned}
\mathbf{j} \cdot \mathbf{J}= & P_{\phi^{\prime}}^{2}-\cot \theta \sin \phi^{\prime} P_{\phi^{\prime}} P_{\theta_{R}^{\prime}}+\frac{\cot \theta}{\sin \theta_{R}^{\prime}} \\
& \times \cos \phi^{\prime} P_{\phi^{\prime}}\left(P_{\phi_{R}^{\prime}}-\cos \theta_{R}^{\prime} P_{\phi^{\prime}}\right) .
\end{aligned}
$$

${ }^{1}$ R. Schriever, M. Chergui, and N. Schwentner, J. Chem. Phys. 93, 9206 (1990).

${ }^{2}$ E. I. Tarasova, A. M. Ratner, V. M. Stepanenko, I. Y. Fugol, M. Chergui, R. Schriever, and N. Schwentner, J. Chem. Phys. 98, 7786 (1993).

${ }^{3}$ R. Schriever, M. Chergui, O. Ünal, and N. Schwentner, J. Chem. Phys. 93, 3245 (1990).

${ }^{4}$ R. Schriever, M. Chergui, H. Kunz, V. Stepanenko, and N. Schwentner, J. Chem. Phys. 91, 4128 (1989).

${ }^{5}$ K. H. Gödderz, N. Schwentner, and M. Chergui, J. Chem. Phys. 105, 451 (1996).

${ }^{6}$ K. H. Gödderz, N. Schwentner, and M. Chergui, Chem. Phys. 209, 91 (1996).

${ }^{7}$ J. G. McCaffrey, H. Kunz, and N. Schwentner, J. Chem. Phys. 96, 2825 (1992).

${ }^{8}$ J. G. McCaffrey, H. Kunz, and N. Schwentner, J. Chem. Phys. 96, 155 (1992).

${ }^{9}$ D. LaBrake, E. T. Ryan, and E. Weitz, J. Chem. Phys. 102, 4112 (1995).

${ }^{10}$ C. J. Bardeen et al., J. Chem. Phys. 106, 8486 (1997).

${ }^{11}$ M. Ovchinnikov and V. A. Apkarian, J. Chem. Phys. 105, 1 (1996).

${ }^{12}$ A. I. Krylov and R. B. Gerber, J. Chem. Phys. 106, 6574 (1997).

${ }^{13}$ A. I. Krylov, R. B. Gerber, and R. D. Coalson, J. Chem. Phys. 105, 4626 (1996).

${ }^{14}$ P. Jungwirth, E. Fredj, and R. B. Gerber, J. Chem. Phys. 104, 9332 (1996).

${ }^{15}$ L. Liu and H. Guo, J. Chem. Phys. 104, 528 (1996).

${ }^{16}$ M. Ben-Num, R. D. Levine, and G. R. Fleming, J. Chem. Phys. 105, 3035 (1996).

${ }^{17}$ M. Ben-Num and R. D. Levine, Chem. Phys. 201, 163 (1995).

${ }^{18}$ J. Manz, P. Saalfrank, and B. Schmidt, J. Chem. Soc., Faraday Trans. 93, 957 (1997).

${ }^{19}$ B. Schmidt, Chem. Phys. Lett. 301, 207 (1999).

${ }^{20}$ P. Zdanska, B. Schmidt, and P. Jungwirth, J. Chem. Phys. 110, 6246 (1999).

${ }^{21}$ I. Nadler, D. Mahgerefteh, H. Reisler, and C. Wittig, J. Chem. Phys. 82, 3885 (1985).

${ }^{22}$ W. P. Hess and S. R. Leone, J. Chem. Phys. 86, 3773 (1987).

${ }^{23}$ O. Atabek, J. A. Beswick, R. Lefebvre, S. Mukamel, and J. Jortner, J. Chem. Phys. 65, 4035 (1976).

${ }^{24}$ M. D. Morse and K. F. Freed, J. Chem. Phys. 70, 3604 (1979).

${ }^{25}$ J. A. Beswick and J. Jortner, Chem. Phys. 24, 1 (1977).

${ }^{26}$ N. E. Henriksen and E. J. Heller, J. Chem. Phys. 91, 4700 (1989).

${ }^{27}$ H. Guo and G. C. Schatz, J. Chem. Phys. 92, 1634 (1990).

${ }^{28}$ Y. Wang and C. X. W. Qian, J. Chem. Phys. 100, 2707 (1994).

${ }^{29}$ J. Vigué, B. Girard, G. Gouédard, and N. Billy, Phys. Rev. Lett. 62, 1358 (1989)

${ }^{30}$ J. M. Bowman and R. C. Mayrhofer, J. Chem. Phys. 101, 9469 (1994).

${ }^{31}$ F. N. Dzegilenko, J. M. Bowman, and Y. Amatatsu, Chem. Phys. Lett. 264, 24 (1997).

${ }^{32}$ Y. Wang and C. X. W. Qian, Chem. Phys. Lett. 219, 389 (1994).

${ }^{33}$ R. W. Heather and J. C. Light, J. Chem. Phys. 78, 5513 (1983).

${ }^{34}$ M. Jacon, P. Guérin, O. Atabek, and H. L. Rouzo, J. Chem. Phys. 97, 2490 (1992)

${ }^{35}$ J. A. Beswick, M. Glass-Maujean, and O. Roncero, J. Chem. Phys. 96, 7514 (1992)

${ }^{36}$ C. J. Williams, J. Quian, and D. J. Tannor, J. Chem. Phys. 95, 1721 (1991).

${ }^{37}$ J. F. Black, J. Chem. Phys. 98, 6853 (1993).

${ }^{38}$ J. F. Black, J. R. Waldeck, and R. N. Zare, J. Chem. Phys. 92, 3519 (1990).

${ }^{39}$ W. Krieger, J. Häger, and J. Pfab, Chem. Phys. Lett. 85, 69 (1982).

${ }^{40}$ J. Quian, D. J. Tannor, Y. Amatatsu, and K. Morokuma, J. Chem. Phys. 101, 9597 (1994).

${ }^{41}$ F. Shokoohi, S. Hay, and C. Wittig, Chem. Phys. Lett. 110, 1 (1984).

${ }^{42}$ E. Hasselbrink, J. R. Waldeck, and R. N. Zare, Chem. Phys. 126, 191 (1988).

${ }^{43}$ J. F. Black, E. Hasselbrink, J. R. Waldeck, and R. N. Zare, Mol. Phys. 71, 1143 (1990).

${ }^{44}$ G. E. Hall, N. Sivakumar, and P. L. Houston, J. Chem. Phys. 84, 2120 (1986).

${ }^{45}$ H. Joswig, M. A. O'Halloran, R. N. Zare, and M. S. Child, Faraday Discuss. Chem. Soc. 82, 79 (1986).

${ }^{46}$ W. M. Pitts and A. P. Baronavski, Chem. Phys. Lett. 71, 395 (1980).

${ }^{47}$ I. Nadler, H. Reisler, and C. Wittig, Chem. Phys. Lett. 103, 451 (1984). 
${ }^{48}$ E. M. Goldfield, P. L. Houston, and G. S. Ezra, J. Chem. Phys. 84, 3120 (1986).

${ }^{49}$ H. Wei and T. Carrington, Jr., J. Chem. Phys. 105, 141 (1996).

${ }^{50}$ M. A. O'Halloran, H. Joswig, and R. N. Zare, J. Chem. Phys. 87, 303 (1987).

${ }^{51}$ M. Dantus, M. J. Rosker, and A. H. Zewail, J. Chem. Phys. 87, 2395 (1987).

${ }^{52}$ M. J. Rosker, M. Dantus, and A. H. Zewail, J. Chem. Phys. 89, 6113 (1988).

${ }^{53}$ M. Dantus, M. J. Rosker, and A. H. Zewail, J. Chem. Phys. 89, 6128 (1988).

${ }^{54}$ S. Yabushita and K. Morokuma, Chem. Phys. Lett. 175, 518 (1990).

${ }^{55}$ Y. Amatatsu, S. Yabushita, and K. Morokuma, J. Chem. Phys. 100, 4894 (1994).

${ }^{56}$ J. C. Tully, J. Chem. Phys. 93, 1061 (1990).

${ }^{57}$ S. Hammes-Schiffer and J. C. Tully, J. Chem. Phys. 101, 4657 (1994).

${ }^{58}$ S. Fernández-Alberti, N. Halberstadt, J. A. Beswick, and J. Echave, J. Chem. Phys. 109, 2844 (1998).

${ }^{59}$ E. J. Heller, J. Chem. Phys. 62, 1544 (1975).

${ }^{60}$ E. J. Heller, J. Chem. Phys. 75, 2923 (1981).

${ }^{61}$ S. M. Anderson, J. I. Zink, and D. Neuhauser, Chem. Phys. Lett. 291, 387 (1998).

${ }^{62}$ G. Parlant and E. A. Gislason, J. Chem. Phys. 91, 4416 (1989).

${ }^{63}$ M. Ben-Num and T. J. Martinez, J. Chem. Phys. 108, 7244 (1998).

${ }^{64}$ R. Fraenkel and Y. Haas, Chem. Phys. Lett. 214, 234 (1993).

${ }^{65}$ U. Samuni, S. Kahana, R. Fraenkel, Y. Haas, D. Danovich, and S. Shaik, Chem. Phys. Lett. 225, 391 (1994).

${ }^{66}$ D. Raftery, E. Gooding, A. Romanovsky, and R. M. Hochstrasser, J. Chem. Phys. 101, 8572 (1994).
${ }^{67}$ A. I. Krylov and R. B. Gerber, J. Chem. Phys. 100, 4242 (1994).

${ }^{68}$ I. Benjamin, J. Chem. Phys. 103, 2459 (1995).

${ }^{69}$ C. Wan, M. Gupta, and A. H. Zewail, Chem. Phys. Lett. 256, 279 (1996).

${ }^{70}$ Y. Amatatsu and K. Morokuma, Chem. Phys. Lett. 245, 469 (1995).

${ }^{71}$ M. Chergui, private communication.

${ }^{72}$ A. Bastida, J. Zuñiga, A. Requena, N. Halberstadt, and J. A. Beswick, J. Chem. Phys. 109, 6320 (1998).

${ }^{73}$ C. H. Becker, P. Casavecchia, Y. T. Lee, R. E. Olso, and W. A. Lester, Jr., J. Chem. Phys. 70, 5477 (1979).

${ }^{74}$ I. H. Gersonde and H. Gabriel, J. Chem. Phys. 98, 2094 (1993).

${ }^{75}$ A. A. Buchachenko and N. F. Stepanov, J. Chem. Phys. 104, 9913 (1996).

${ }^{76}$ V. S. Batista and D. F. Coker, J. Chem. Phys. 105, 4033 (1996).

${ }^{77}$ Y. Zhao, I. Yourshaw, G. Reiser, C. C. Arnold, and D. M. Neumark, J. Chem. Phys. 101, 6538 (1994).

${ }^{78}$ M. P. Allen and D. J. Tildesley, Computer Simulations of Liquids (Oxford University Press, Oxford, 1987).

${ }^{79}$ R. Heather and H. Metiu, J. Chem. Phys. 86, 5009 (1987).

${ }^{80}$ T. N. Truong, J. J. Tanner, P. Bala, J. A. McCammon, B. Lesyng, and D. K. Hoffman, J. Chem. Phys. 96, 2077 (1992).

${ }^{81}$ M. Braun, C. Meier, and V. Engel, Comput. Phys. Commun. 93, 152 (1996).

${ }^{82}$ Z. Bačić and J. C. Light, J. Chem. Phys. 85, 4594 (1986).

${ }^{83}$ P. Pernot and W. A. Lester, Int. J. Quantum Chem. 40, 577 (1991).

${ }^{84}$ R. Schinke, Photodissociation Dynamics (Cambridge University Press, New York, 1993).

${ }^{85}$ R. N. Zare, Angular Momentum: Understanding Spatial Aspects in Chemistry and Physics (Wiley, New York, 1988).

${ }^{86}$ H. Goldstein, Classical Mechanics (Addison-Wesley, New York, 1950).

${ }^{87}$ G. C. Schatz and A. Kuppermann, J. Chem. Phys. 65, 4642 (1976). 